\title{
ABCG2 downregulation in glioma stem cells enhances the therapeutic efficacy of demethoxycurcumin
}

\author{
Long Chen ${ }^{1,2, *}$, Lei Shi ${ }^{3, *}$, Wenhua Wang ${ }^{2, *}$ and Youxin Zhou ${ }^{1}$ \\ ${ }^{1}$ Department of Neurosurgery, The First Affiliated Hospital of Soochow University, Suzhou 215006, P. R. China \\ ${ }^{2}$ Department of Neurosurgery, Traditional Chinese Medicine Hospital of Kunshan, Affiliated Nanjing University of Traditional \\ Chinese Medicine, Suzhou 215300, P. R. China \\ ${ }^{3}$ Department of Neurosurgery, The First People's Hospital of Kunshan Affiliated with Jiangsu University, Suzhou 215300, \\ P. R. China \\ *These authors have contributed equally to this work \\ Correspondence to: Lei Shi, email: s11012002322@126.com \\ Youxin Zhou, email: zhouyxyq2008@sohu.com \\ Keywords: glioma stem cells, demethoxycurcumin, reactive oxygen species, caspase-3, ABCG2 \\ Received: February 20, $2017 \quad$ Accepted: April 06, $2017 \quad$ Published: May 19, 2017 \\ Copyright: Chen et al. This is an open-access article distributed under the terms of the Creative Commons Attribution License 3.0 (CC BY \\ 3.0), which permits unrestricted use, distribution, and reproduction in any medium, provided the original author and source are credited.
}

\section{ABSTRACT}

We analyzed the role of ABCG2, a drug transporter, in determining the sensitivity of glioma stem cells (GSCs) to demethoxycurcumin (DMC). We first demonstrated that ABCG2 is more highly expressed in GSCs than primary astrocytes. Modulation of ABCG2 levels in GSCs by transfection of ABCG2 shRNA or a lentiviral vector encoding ABCG2 revealed an inverse relation between ABCG2 levels and DMC-induced GSC growth inhibition. Suppressing ABCG2 increased DMC-induced apoptosis and G0/G1 cell cycle arrest in GSCs. It also increased levels reactive oxygen species (ROS) in GSCs treated with DMC, resulting in increased cytochrome $C$ and caspase- 3 activity. When GSCs transfected with ABCG2 shRNA or overexpressing ABCG2 were xenografted and the tumor-bearing, immunodeficient mice were treated with DMC, ABCG2 expression suppressed the tumor proliferation rate (T/C \%). These findings demonstrate that ABCG2 expression is critical for DMC resistance in GSCs and is a potential therapeutic target for GBM.

\section{INTRODUCTION}

Glioma stem cells (GSCs) are responsible for the recurrence of glioblastoma multiforme (GBM) and insensitivity to Temozolomide (TMZ), the first-line drug for GBM treatment. GBM is an aggressive type of malignant glioma with very low 2- and 5-year survival outcomes [1]. There are no effective agents to treat GSCs and the efficacy of adjuvant therapy is limited. TMZ is a 3-methyl derivative of mitozolomide that forms $\mathrm{O}^{6}$-methylguanine, which inhibits GBM cell proliferation and induces apoptosis and autophagy in glioma cells [2]. However, TMZ is ineffective in inhibiting GSC proliferation and inducing their apoptosis [3]. Fueyo et al. suggested that $\mathrm{O}^{6}$-methyl guanine -DNA methyl transferase (MGMT) was responsible for resistance of glioma cells to TMZ and therefore MGMT expression formed the basis for clinical treatment strategies. However, recent studies showed that drug resistance in GSCs was related to ABCG2 and not MGMT [4]. This suggested that the mechanisms of drug resistance in glioma cells and GSCs were different.

ATP-binding cassette sub-family $G$ member 2 (ABCG2) is a xenobiotic transporter that confers resistance to a variety of anticancer drugs by transporting intracellular drugs out of tumor cells. Robey et al found that overexpression of $\mathrm{ABCG} 2$ lowered intracellular levels of photosensitizers below the threshold required to induce significant tumor cell death [5]. Inhibition of the ABCG2 transporter improved the efficacy of photodynamic therapy on keratinocytes [6]. Recent studies showed that ABCG2 expression was partly responsible for increased resistance of GSCs to chemotherapy. Jia et al. reported that inhibition of $\mathrm{ABCG} 2$ sensitized $\mathrm{CD} 90^{+} \mathrm{CD} 133^{+}$liver $\mathrm{CSCs}$ to chemotherapeutic agents [7]. ABCG2 was also highly expressed in breast cancer stem cells (BCSCs) and its inhibition by 5 -aza-2'-deoxycytidine (DAC) sensitized BCSCs to doxorubicin, verapamil, and tamoxifen [8]. 
Also, cervical cancer stem cells (CSCs), which are characterized by prolonged cell survival, infinite cell proliferation and highly resistant apoptosis became sensitized by silencing ABCG2 expression [9]. Recently, ABCG2 overexpression was confirmed in all GSCs [10, 11]. Xu et al reported that high expression of $\mathrm{ABCG} 2$ in GSCs reduced accumulation of chemotherapeutic agents and resulted in drug resistance [11]. Also, inhibition of ABCG2 improved the efficacy of sonodynamic therapy (SDT) in GSCs [11]. Jin et al reported that high ABCG2 expression in $\mathrm{CD}_{133^{+}}$GSCs conferred mitoxantone resistance [12].

Demethoxycurcumin (DMC) is a major component of Curcuma longa L, which effectively inhibited proliferation and induced apoptosis in GSCs in vitro [13]. However, its mechanism of action is not fully understood. Therefore, in the current study, we investigated the role of ABCG2 in the chemoresistance of GSCs to DMC and if its downregulation improved therapeutic efficacy of DMC in a mouse xenograft model.

\section{RESULTS}

\section{ABCG2 expression in primary astrocytes and GSCs}

Previous research showed that 40-50\% WHO III and WHO IV glioma tissues and 100\% U251 GSCs overexpressed ABCG2 [11, 12]. Hence, we analyzed ABCG 2 expression in primary astrocytes and GSCs by RT-PCR and western blotting. As shown in Figure 1A and $1 \mathrm{~B}$, we observed high mRNA and protein expression of ABCG2 in the primary GSCs and no expression in the primary astrocytes. Further, immunohistochemical staining of GSC spheres (Figure 1C) and flow cytometry analysis showed that more than 97\% GSC sphere cells were ABCG2-positive (Figure 1B). These results demonstrated that $\mathrm{ABCG} 2$ was highly expressed in the GSCs and probably played an important role in their function.

\section{Association between ABCG2 expression and efficiency of DMC inhibition of GSCs in vitro}

Next, we determined the effects of DMC on cell viability of GSCs by MTT assay. As shown in Figure $2 \mathrm{~A}$, treatment with $10 \mu \mathrm{M}$ DMC resulted in cell growth inhibition of GSCs by $3.7 \%, 7.3 \%$ and $11.6 \%$ at 24,48 and $72 \mathrm{~h}$, respectively. Similarly, 30 $\mu$ M DMC inhibited cell growth of GSCs by $7.3 \%, 15.3 \%$, and $23.6 \%$ at 24,48 and $72 \mathrm{~h}$, respectively.

Further, we investigated if ABCG2 expression influenced DMC-induced GSC growth inhibition. Towards this, we transfected GSCs with lenti-GFPABCG2 shRNA and determined that ABCG2 was significantly downregulated in GSCs (Figure 2B). Then, we tested the inhibitory efficiency of DMC in ABCG2 knockdown GSCs. As shown in Figure 2C, treatment of ABCG2 knockdown GSCs with $10 \mu \mathrm{M}$ DMC showed growth inhibition of $13.2 \%, 23.7 \%$ and $31.6 \%$ for GSC1 and $7.2 \%, 15.3 \%$, and $23.6 \%$ at for GSC-2 at 24,48 and $72 \mathrm{~h}$, respectively. When treated with $30 \mu \mathrm{M} \mathrm{DMC}$, the ABCG2 knockdowns GSC1 and GSC-2 showed a growth inhibition rate of $15.3 \%, 27.1 \%$, and $47.3 \%$ and $9.7 \%$,

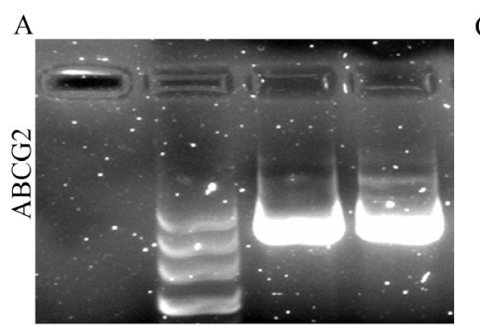

astrocytes Marker GSC-1 GSC-2

$$
\text { B }
$$

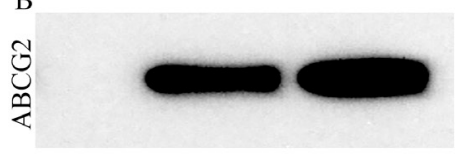

astrocytes $\quad$ GSC-1

GCS-2

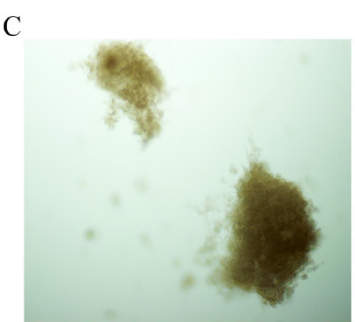

GSC-1

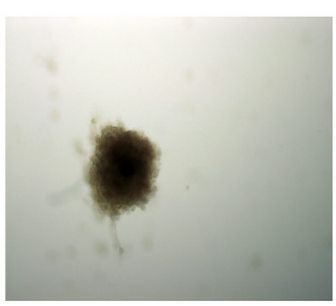

GSC-2

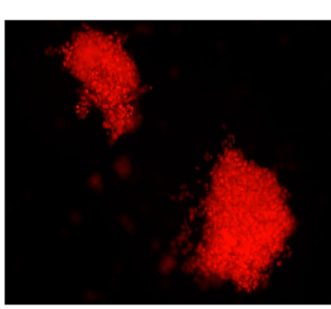

GSC-1: ABCG2

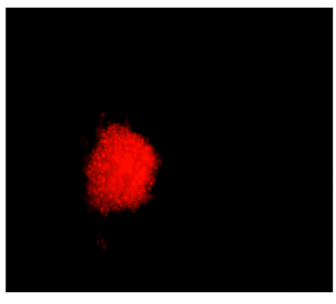

GSC-2:ABCG2
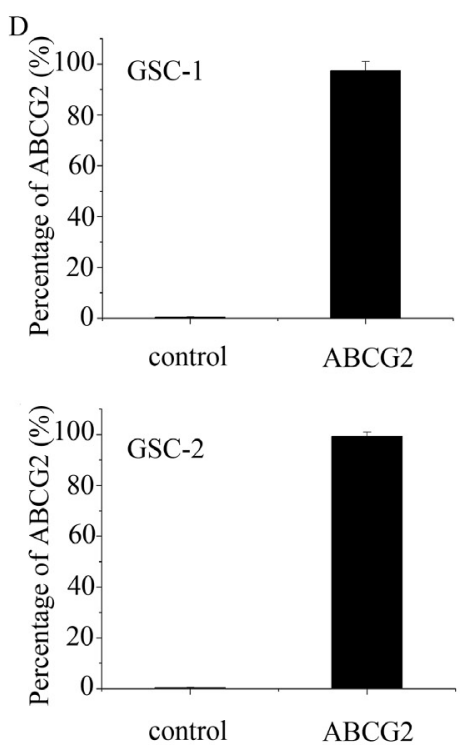

Figure 1: The expression of ABCG2 in the primary astrocytes and GSCs. (A, B) ABCG2 mRNA and protein levels in primary GSCs as detected by RT-PCR and Western blot, respectively. (C) Immunohistochemical analysis showing ABCG2 expression in GSC spheres. (D) Flow cytometry analysis of ABCG2 expression in GSC spheres. 

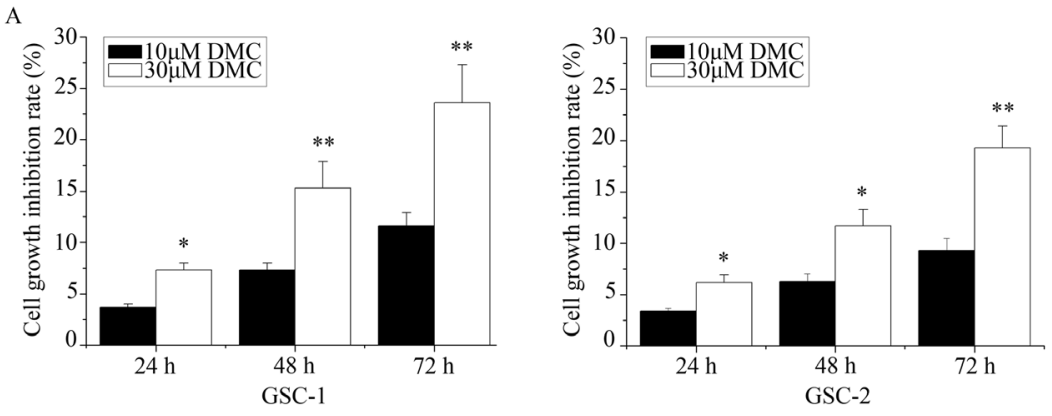

B
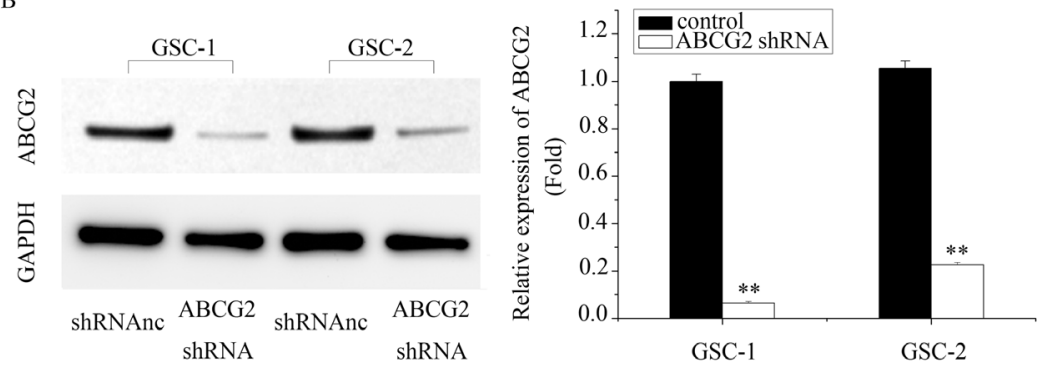

$\mathrm{C}$
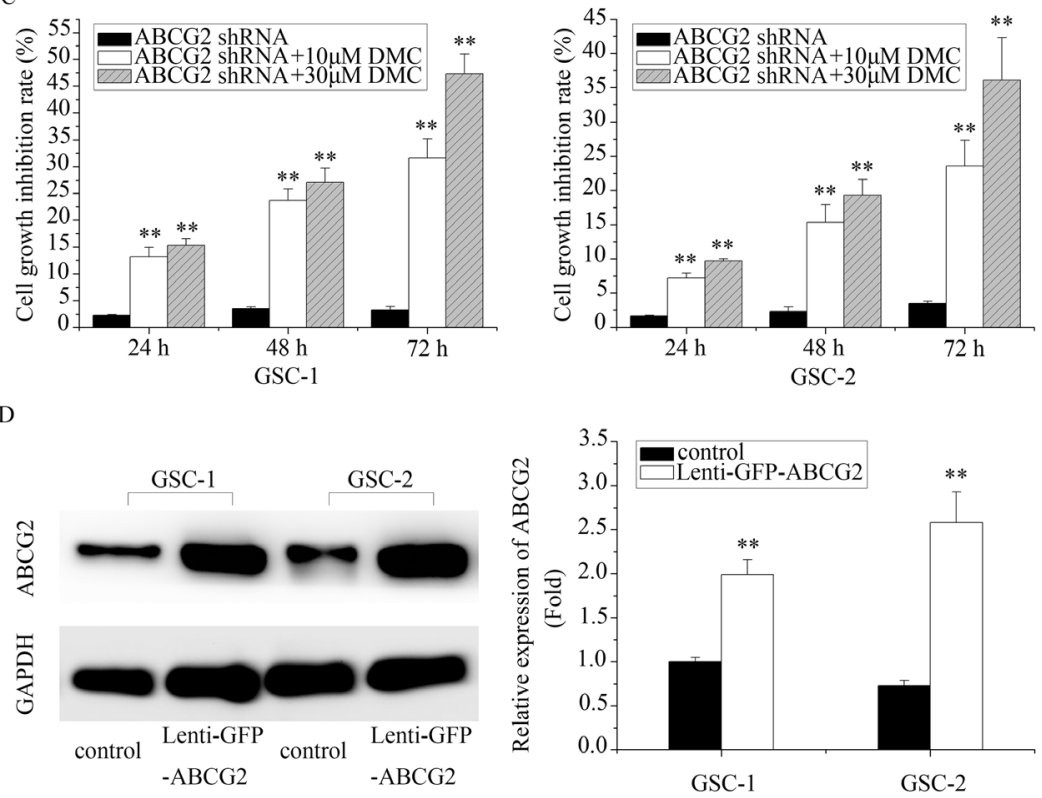

$\mathrm{E}$
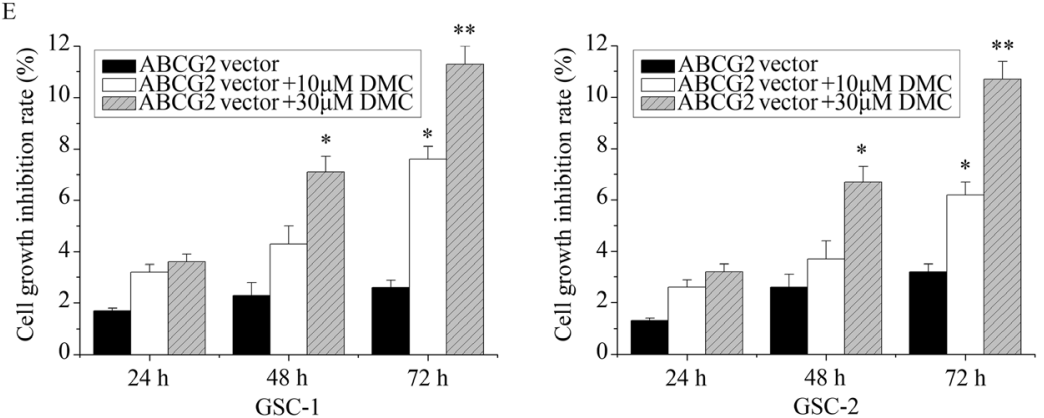

Figure 2: The in vitro effects of differential ABCG2 expression on DMC inhibition of GSCs. (A) The cell growth inhibitory effects of $10 \mu \mathrm{M}$ or 30 $\mu \mathrm{M}$ DMC on GSCs as measured by MTT assay. (B) Western blot analysis of ABCG2 expression in GSCs transfected

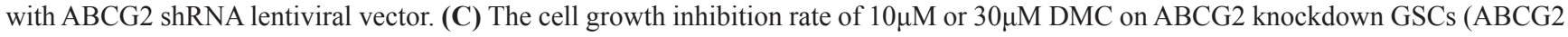
shRNA) as determined by MTT assay. (D) Western blot analysis of ABCG2 expression in GSCs transfected with ABCG2 overexpression lentiviral vector. (E) The cell growth inhibition rate of $10 \mu \mathrm{M}$ or $30 \mu \mathrm{M}$ DMC on ABCG2 overexpressed GSCs as determined by MTT assay. "Lenti-GFP-ABCG2" is denoted as "ABCG vector"."Lenti-GFP-ABCG2 shRNA" is denoted as "ABCG2 shRNA". 
$19.3 \%$ and $36.1 \%$ at $24,48,72 \mathrm{~h}$, respectively. Conversely, we transfected GSCs with ABCG2 overexpressed vector (lenti-GFP-ABCG2) and tested the growth inhibition effects of 10 or $30 \mu \mathrm{M}$ DMC in GSC-1 and GSC-2. As shown in Figure 2D, we observed increased resistance to $\mathrm{DMC}$ in $\mathrm{ABCG} 2$ overexpressed GSC-1 and GSC2 compared to the controls. Collectively, these data suggested that ABCG2 expression levels inversely correlated with DMC efficacy in inhibiting GSCs.

\section{Evaluation of ABCG2 expression on the anti- GSC effects of DMC in vivo}

Next, we evaluated the in vivo relevance of high or low ABCG2 expression on the DMC inhibition of GSCs by implanting $10^{6} \mathrm{CD} 133$-positive GSCs transfected with either ABCG2 shRNA or overexpression lentiviral vectors into immune-deficient nude mice. When the tumor volume reached about $50 \mathrm{~mm}^{3}$, the xenograft tumor-bearing nude mice were administered with either $10 \mathrm{mg} / \mathrm{kg}$ or $30 \mathrm{mg} /$ $\mathrm{kg}$ DMC. After 30 days, the relative tumor proliferation rate $\mathrm{T} / \mathrm{C}(\%)$ was determined to evaluate the antitumor activity of DMC as described in the methods. As shown in Figure $3 \mathrm{~A}, \mathrm{~T} / \mathrm{C}(\%)$ in $10 \mathrm{mg} / \mathrm{kg}$ or $30 \mathrm{mg} / \mathrm{kg}$ DMC-alone treatment group was $43.61 \%$ and $35.72 \%$ for GSC-1 and $53.61 \%$ and $37.62 \%$ for GSC-2, respectively. The T/C (\%) for ABCG2 knockdown (lenti-GFP-ABCG2 shRNA) GSCs was $30.61 \%$ and $23.71 \%$ for GSC- 1 and $43.71 \%$ and $29.31 \%$ for GSC-2, respectively for the $10 \mathrm{mg} / \mathrm{kg}$ or $30 \mathrm{mg} /$ $\mathrm{kg}$ DMC groups. These data suggested that downregulation of ABCG2 enhanced the anti-tumor activity of DMC on the GSC xenograft tumors. Conversely, the T/C (\%) for ABCG2 overexpression (lenti-GFP-ABCG2) GSCs was 63.21\% and $53.21 \%$ in GSC-1 and $67.62 \%$ and $55.36 \%$ in GSC2 , respectively for the $10 \mathrm{mg} / \mathrm{kg}$ or $30 \mathrm{mg} / \mathrm{kg}$ DMC groups.
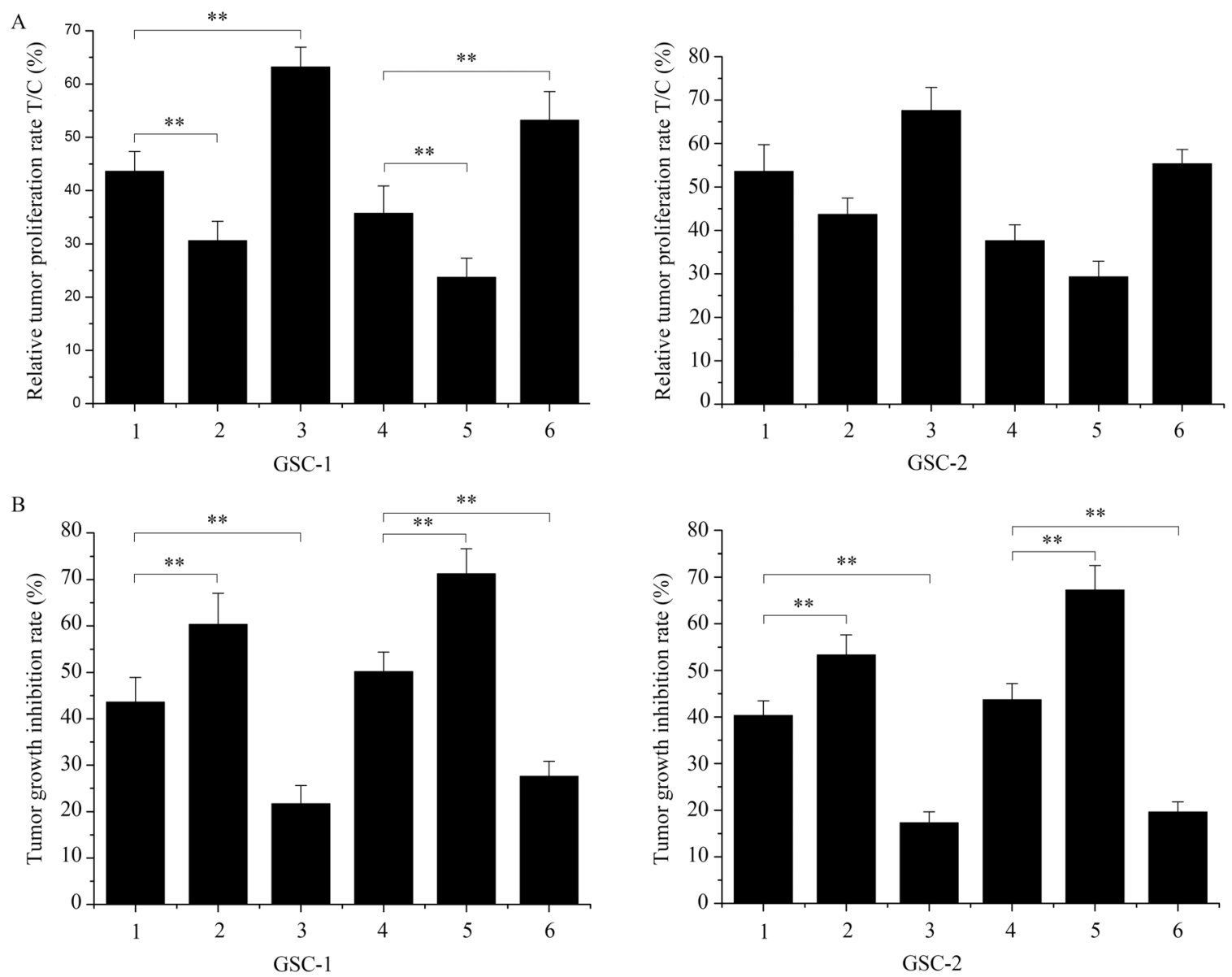

1:10mg/kg DMC; $2: 10 \mathrm{mg} / \mathrm{kg}$ DMC+Lenti-GFP-ABCG2 shRNA; 3:10 $\mathrm{mg} / \mathrm{kg}$ DMC+Lenti-GFP-ABCG2;

4:30mg/kg DMC; 5:30mg/kg DMC+Lenti-GFP-ABCG2 shRNA; 6:30mg/kg DMC+Lenti-GFP-ABCG2

Figure 3: In vivo effects of DMC on GSCs with low or high ABCG2 expression. Balb/c nude mice were xenografted with GSCs that were transfected with either lenti-GFP-ABCG2 shRNA or lenti-GFP-ABCG2 to downregulate or overexpress ABCG2 in GSCs, respectively. After 30 days, the mice were administered $10 \mathrm{mg} / \mathrm{kg}$ or $30 \mathrm{mg} / \mathrm{kg} \mathrm{DMC}$ for 30 days. Then, relative tumor proliferation rates $\mathrm{T} / \mathrm{C}$ $\%$ (A) and the tumor growth inhibition rates (TGI \%) (B) were calculated. 
This suggested that ABCG2 overxpression decreased the anti-tumor activity of DMC on GSCs xenograft tumors.

Further, tumor growth inhibition rate (\%) (TGI\%) in $10 \mathrm{mg} / \mathrm{kg}$ or $30 \mathrm{mg} / \mathrm{kg}$ DMC-alone treatment group was $43.62 \%$ and $50.16 \%$ in GSC- 1 and $40.32 \%$ and $43.71 \%$ in GSC-2, respectively, whereas ABCG2 knockdown (LentiGFP-ABCG2 shRNA) resulted in the TGI\% for the $10 \mathrm{mg} /$ $\mathrm{kg}$ or $30 \mathrm{mg} / \mathrm{kg} \mathrm{DMC}$ groups was $60.32 \%$ and $71.23 \%$ in GSC- 1 and $53.32 \%$ and $67.21 \%$ in GSC-2, respectively. In contrast, $\mathrm{ABCG} 2$ overxpression (Lenti-GFP-ABCG2) resulted in the TGI $\%$ for the $10 \mathrm{mg} / \mathrm{kg}$ or $30 \mathrm{mg} / \mathrm{kg} \mathrm{DMC}$ group were $21.70 \%$ and $27.63 \%$ in GSC-1 and $17.32 \%$ and $19.61 \%$ in GSC-2, respectively. These data again showed that $\mathrm{ABCG} 2$ expression negatively regulated the tumor growth inhibition rate of DMC on GSCs in vivo.

\section{Evaluation of ABCG2 expression on cell cycle and apoptosis of DMC on the anti-GSC effects}

Next, we used flow cytometry to determine if altering ABCG2 levels in GSCs resulted in cell cycle changes upon DMC treatment. Treatment of GSCs to
$10 \mu \mathrm{M}$ and $30 \mu \mathrm{M}$ DMC for $48 \mathrm{~h}$ increased $\mathrm{G} 0 / \mathrm{G} 1$ cells proportionately, compared to the controls $(\mathrm{P}<0.05$; Figure 4A). Moreover, ABCG2 downregulation (lentiGFP-ABCG2 shRNA) further increased G0/G1 phase cells and ABCG2 overexpression (lenti-GFP-ABCG2) lowered G0/G1 phase cells upon treatment of GSCs by $10 \mu \mathrm{M}$ and $30 \mu \mathrm{M}$ DMC. These data suggested that ABCG2 expression levels influenced DMC's ability to inhibit cell cycle in the GSCs.

In Figure 4A, we also observed increased sub-G1 upon $30 \mu \mathrm{M}$ DMC treatment of ABCG2 downregulated (lenti-GFP-ABCG2 shRNA) GSCs, suggesting enhanced apoptosis. To confirm enhanced apoptosis upon ABCG2 downregulation, we treated $\mathrm{ABCG} 2$ downregulated GSCs with $30 \mu \mathrm{M}$ DMC and analyzed apoptosis by TUNEL and Histone-DNA ELISA assays. We found that co-administration of lenti-GFP-ABCG2 shRNA and $30 \mu \mathrm{M}$ DMC for $48 \mathrm{~h}$ increased cell apoptosis of $12.15 \%$ in GSCs- 1 and $11 \%$ in GSCs- 2 based on TUNEL assays (Figure 4B), and 0.28-fold in GSCs-1 and 0.2-fold in GSCs-2 based on Histone-DNA ELISA assays (Figure $4 \mathrm{C}$ ) compared with $30 \mu \mathrm{M}$ DMC treatment. These results
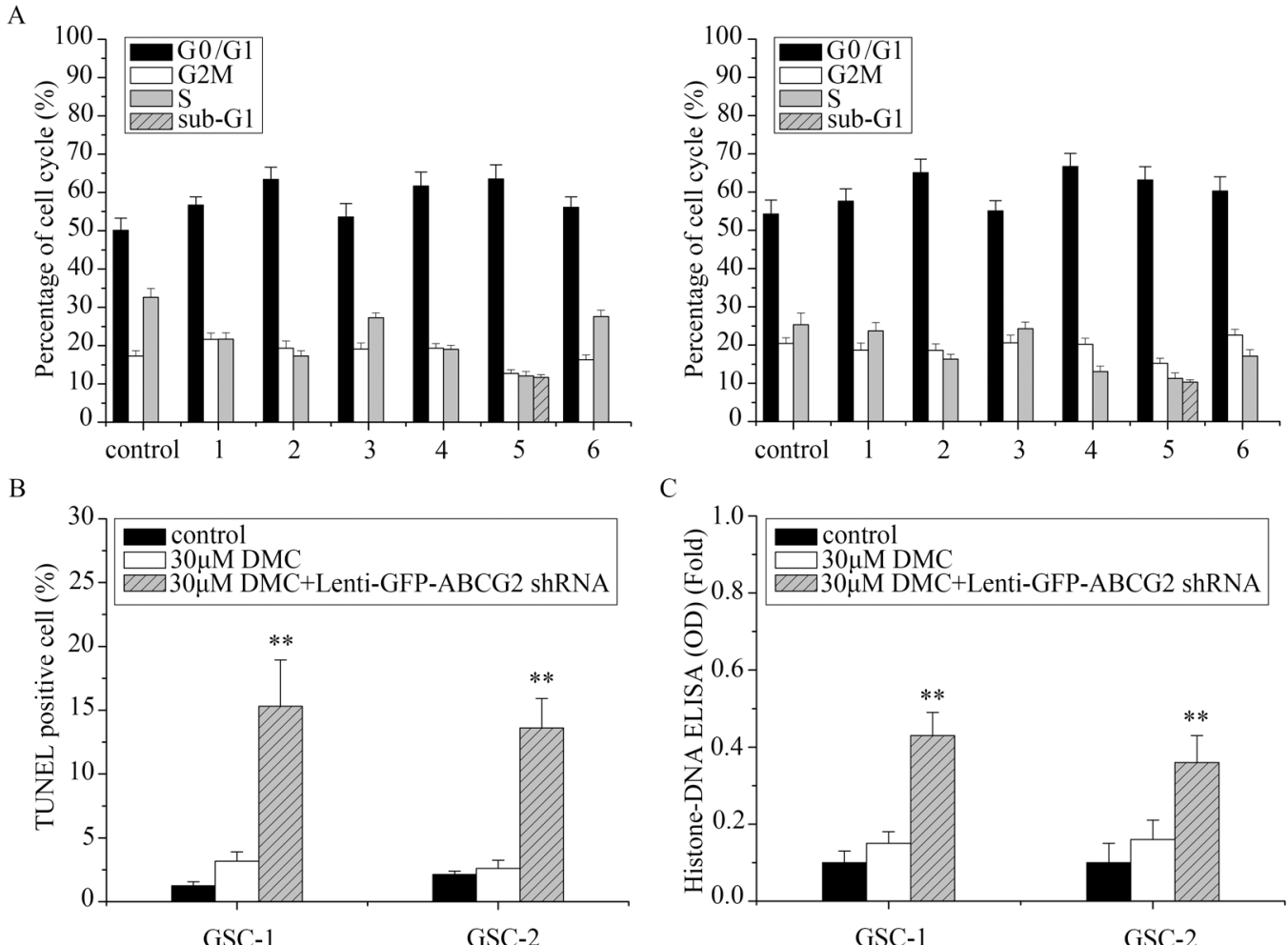

$\mathrm{C}$

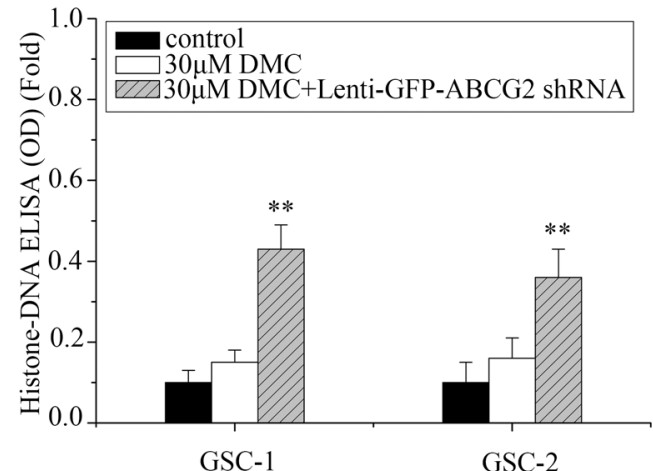

1:10mg/kg DMC; 2:10mg/kg DMC+Lenti-GFP-ABCG2 shRNA; 3:10mg/kg DMC+Lenti-GFP-ABCG2; 4:30mg/kg DMC; 5:30mg/kg DMC+Lenti-GFP-ABCG2 shRNA; 6:30mg/kg DMC+Lenti-GFP-ABCG2

Figure 4: Analysis of cell cycle and apoptosis of GSCs with low or high ABCG2 expression upon DMC treatment. (A) GSCs were transfected with either lenti-GFP-ABCG2 shRNA or lenti-GFP-ABCG2 to downregulate or overexpress ABCG2 in GSCs and treated with either $10 \mu \mathrm{M}$ or $30 \mu \mathrm{M}$ DMC for $24 \mathrm{~h}$ followed by PI staining and flow cytometry analysis of the cell cycle. (B) TUNEL staining to determine apoptosis of GSCs transfected with or without lenti-GFP-ABCG2 shRNA and treated with 30 $\mu$ M DMC. (C) Histone-DNA ELISA analysis of apoptosis in GSCs tranfected with or without lenti-GFP-ABCG2 shRNA and treated with $30 \mu \mathrm{M}$ DMC. 
suggested that downregulation of ABCG2 enhanced DMC induced apoptosis in GSCs.

\section{Effects of $\mathrm{ABCG} 2$ deficiency on enhancing DMC-induced anti-GSCs was mediated by ROS production and caspase- 3 signaling cascade activation}

Recently, Shen et al. reported that ABCG2 protected against oxidative stress by decreasing ROS generation and enhancing antioxidant capacity [14]. Also, Oliva et al showed that low ROS production and tighter mitochondrial coupling influence chemo-resistance to TMZ in glioma [15]. Further, we previously showed that DMC inhibited ROS production [13]. Thus, we postulated that the higher ROS production would increase the inhibitory activity of DMC in ABCG2 downregulated GSCs. As shown in Figure 5A, 30 $\mu \mathrm{M}$ DMC treatment enhanced ROS in ABCG2 downregulated (Lenti-GFPABCG2 shRNA) GSCs compared to control. In contrast, when GSCs with ABCG2 overexpression were treated with $30 \mu \mathrm{M}$ DMC, ROS levels were lower than the control. These results suggested that overexpression of ABCG2 increased antioxidant capacity of GSCs to DMC whereas downregulation of $\mathrm{ABCG} 2$ decreased the antioxidant capacity to DMC.

Further, we treated ABCG2 downregulated GSCs with the antioxidant $\mathrm{N}$-acetyl-L-cysteine (NAC) to block increased ROS induced by $30 \mu \mathrm{M}$ DMC treatment and observed that it resulted in decreased cell growth inhibition rate suggesting that $\mathrm{ABCG} 2$ expression modulated ROS levels that were necessary for growth inhibitory effects of DMC on GSCs (Figure 5B).

Since ROS production activates caspase-3 apoptotic signaling cascade [16], we analyzed the cytochrome c (Cyt c) release and caspase-3 activation after $30 \mu \mathrm{M}$ DMC treatment in ABCG2 downregulated GSCs compared to controls. As shown in Figure 5C and 5D, downregulation of ABCG2 enhanced Cyt c levels and caspase-3 activity. In contrast, overxpression of $\mathrm{ABCG} 2$ reduced Cyt $\mathrm{c}$ (Figure 5C) and caspase-3 activity (Figure 5D) upon DMC treatment. These data suggested that ABCG2 levels influenced DMC induced ROS-dependent activation of caspase-3 mediated apoptosis in GSCs.

\section{DISCUSSION}

In this study, we confirmed that ABCG2 expression in GSCs determined the efficacy of DMC. GSCs are resistant to most chemotherapeutic agents. In glioma cells, drug resistance for TMZ was related to O6- methyl guanine -DNA- methyl transferase (MGMT) expression [17]. However, recent studies have shown that high ABCG2 expression resulted in poor therapeutic effects of TMZ on GSCs [4]. It was noted that the high $\mathrm{ABCG} 2$ transporter protein levels increased GSC resistance to chemotherapeutic drugs [11]. ABCG2 pumps various chemotherapeutic drugs out of the cells utilizing ATP hydrolysis and resulted in the drug resistance manifestation of the GSCs [18]. Thus, it was postulated that the $\mathrm{ABCG} 2$ levels were related to chemotherapeutic efficacy.

Curcuminoids are yellow and slightly acidic diarylheptanoids that include curcumin and its derivates demethoxycurcumin (DMC) and bisdemethoxycurcumin (BDMC) [19]. All three curcuminoids inhibit tumor cell division and proliferation, with BMDC showing strongest biological activity. However, recent studies have shown that DMC is most effective against gliomas [20]. Many studies have reported that the three monomers of curcuminoids reverse the overexpression of $\mathrm{ABC}$ transporters including $\mathrm{ABCB} 1, \mathrm{ABCG} 2$, and $\mathrm{ABCC} 1$ in drug resistant tumor cells without causing systemic toxicity [21, 22]. In our previous research, we demonstrated that DMC potently inhibited proliferation and induced apoptosis of GSCs [23]. Thus, we postulated that ABCG2 was involved in drug resistance of GSCs to DMC.

To explore the role of ABCG2 on modulating the chemosensitivity of GSCs, we downregulated or overexpressed ABCG2 in GSCs and tested the effects of $\mathrm{DMC}$ in vitro and in vivo. We observed that $\mathrm{ABCG} 2$ was overexpressed in most of $\mathrm{CD} 133^{+}$GSCs and its downregulation resulted in increased inhibition of GSC proliferation and enhanced apoptosis by DMC. In contrast, overexpression of ABCG2 in GSCs decreased the sensitivity of GSCs to DMC. Thus, our data demonstrated that $\mathrm{ABCG} 2$ represented a more attractive therapy target for GSC chemotherapy.

Low intracellular levels of ROS are critical for normal cell signaling function. However, over production of ROS damages cellular components including proteins, lipids and DNA, which ultimately results in cell death [24]. ROS also play a crucial role in diverse processes of various cancers. Cancer cells often display higher ROS levels that promote cancer cell growth and progression, although it also renders cancer cells more vulnerable to adverse effects of high ROS. In fact, most chemotherapeutic agents kill cancer cells by inducing oxidative stress. Buranrat et al. reported that simvastatin and atorvastatin inhibited cancer cell proliferation and induced apoptosis by increasing ROS in KKU-100 cell line of human cholangiocarcinoma [25]. In leukemia cells, docosahexaenoic acid sensitized leukemic lymphocytes to barasertib and everolimus by enhancing ROS and strongly inducing apoptosis [26]. In colon cancer, arctigenin induced apoptosis through increased ROS by activation of the p38 MAPK pathway [27]. Our previous research showed DMC increase ROS in GSCs and triggered a robust increase in GSC apoptosis [23]. 
A
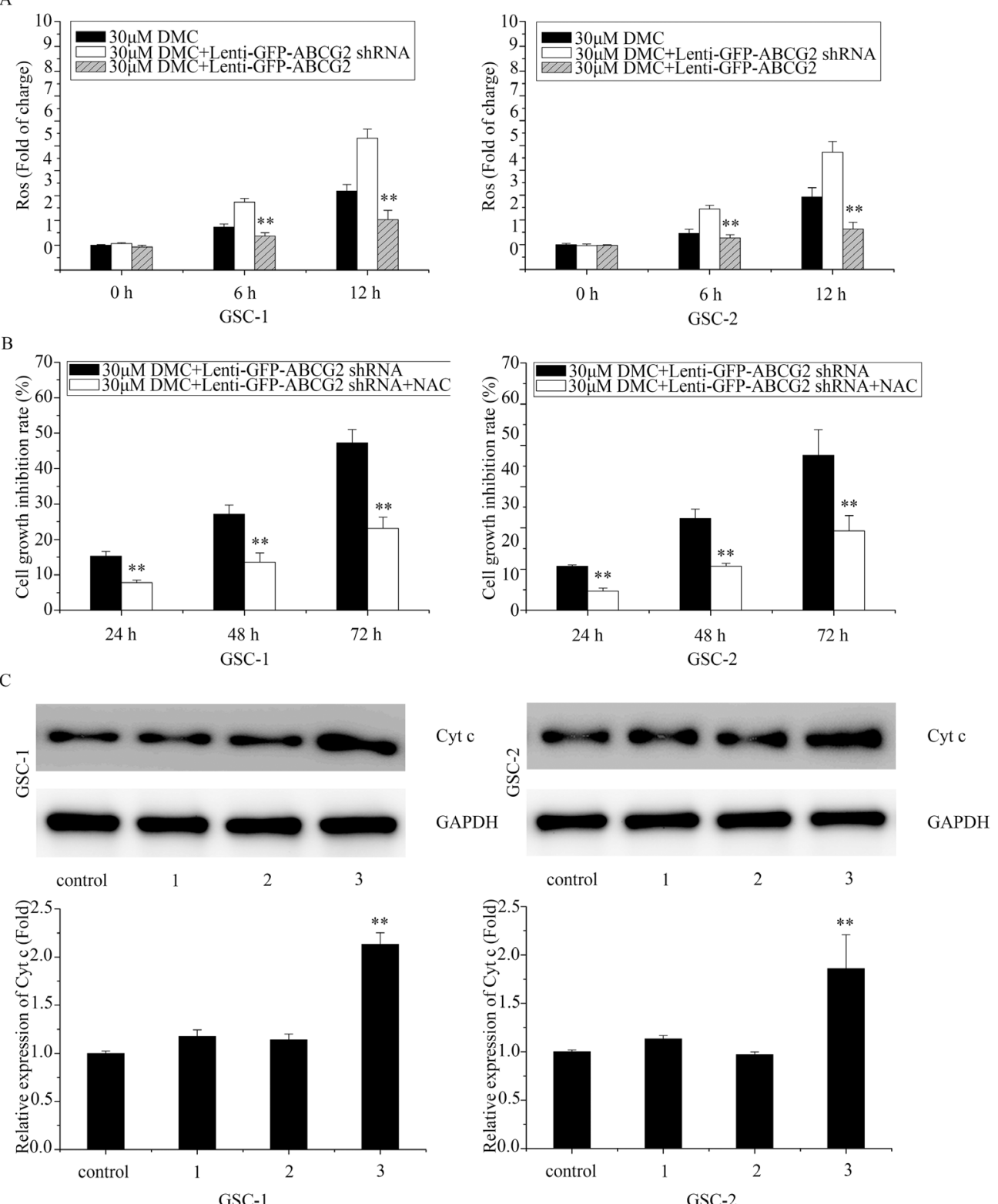

D
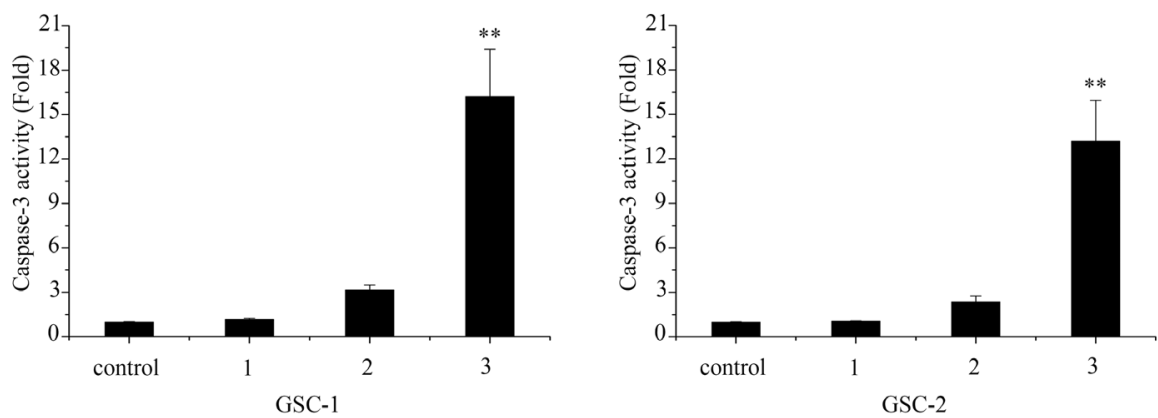

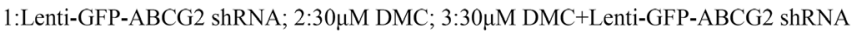

Figure 5: Effects of ABCG2 knockdown on ROS and apoptotic mechanisms upon DMC treatment. (A) FACS analysis

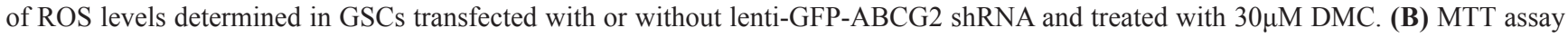
analyzing cell growth inhibition rate in GSCs transfected with lenti-GFP-ABCG2 shRNA and treated with $30 \mu \mathrm{M}$ DMC in presence or absence of 10mM NAC. (C) Western blot analysis of Cyt $\mathrm{c}$ in GSCs transfected with or without lenti-GFP-ABCG2 shRNA and treated with $30 \mu \mathrm{M}$ DMC. (D) Caspase-3 activity assay in GSCs transfected with or without lenti-GFP-ABCG2 shRNA and treated with 30 $\mu \mathrm{M}$ DMC. 
However, the molecular pathways involved in DMCinduced ROS that result in cancer cell apoptosis have remained unclear. Recently, Shen et al. found that ABCG2 played a protective role against oxidative stress by decreasing ROS generation and enhancing antioxidant capacity in Alzheimer's disease [28]. They showed that ABCG2 overexpression inhibited ROS activation, protected cells from ROS-induced toxicity/death, and the downregulation of ABCG2 lead to increased ROS and inflammatory response. Also, ABCG2 exported cytosolic oxidative molecules and reduced overall cellular oxidative capacity. Thus, we postulated that the selective increase of cellular antioxidant capacity may be one of the possible mechanisms by which ABCG2 protected GSCs against DMC. Our data demonstrated that either upregulation or downregulation of ABCG2 in GSCs resulted in lower or higher levels of ROS, respectively, confirming our hypothesis. Therefore, our study demonstrated that DMC induced oxidative stress in cancer cells and ABCG2 overexpression decreased ROS, thereby reducing the efficacy of DMC in GSCs. We demonstrated that knockdown of ABCG2 restored high ROS levels and therefore enhanced sensitivity of GSCs to DMC. Ding et al reported that increased ROS resulted in extensive oxidative DNA damage that upregulated pro-apoptotic Bax and cleaved caspase-3, implying that increased ROS activated caspase-3 signaling [29]. Therefore, we analyzed the expression of Bax, Cyt c and caspase- 3 and confirmed that DMC treatment in GSCs with ABCG2 knockdown increased Cyt c levels and caspase-3 activity whereas GSCs with ABCG2 overexpression decreased Cyt c levels and caspase-3 activity during DMC treatment.

In summary, we demonstrated that modulating the expression of ABCG2 altered the efficacy of DMC therapy on GSCs by regulating ROS production and thereby influencing the caspase-3- apoptotic pathway. Hence, ABCG2 is critical for DMC resistance in GSCs and is a potential therapeutic target for GBM.

\section{MATERIALS AND METHODS}

\section{Primary glioblastoma cell cultures}

Human glioblastoma tissues were obtained after informed consent from 2 adult patients diagnosed with WHO grade IV glioma. Primary glioblastoma cell cultures were obtained after mechanical dissociation according to the protocol described by Darling et al [30]. The cells were subcultured in DMEM with $2 \%$ fetal calf serum (FCS) to prevent the growth of contaminating rodent fibroblasts for 1 week after which the cells were cultured in $10 \%$ FCS and antibiotics of Penicillin and Streptomycin. Glial origin was confirmed by morphology and staining with the anti-glial fibrillary acidic protein (GFAP) monoclonal antibody (mAb) clone 6F2 (Dako, Glostrup, Denmark). All experiments on these cells were performed before passage 5 .

\section{Magnetic cell separation of CD133-positive cells}

Primary gliostoma cells were dissociated from cell culture dishes and resuspended in PBS with $0.5 \%$ BSA and 2mM EDTA and incubated with CD133/1 microbeads (Miltenyi Biotech) followed by positive magnetic cell separation using several MACS columns. The purified $\mathrm{CD}_{133^{+}}$cells were stained with antiCD133/2-PE (Miltenyi Biotech) and analyzed on a BD FACSCalibur. Then, neurosphere cultures were obtained by growing $\mathrm{CD} 133^{+}$tumor cells in stem cell-permissive DMEM/F12 medium supplemented with $20 \mathrm{ng} / \mathrm{ml}$ each of human recombinant epidermal growth factor and human recombinant basic fibroblast growth factor ( $R$ and D Systems), and human leukemia inhibitory factor (Chemicon) and 2\% B27 (Life Technologies). These culture conditions enabled tumor cells to retain the molecular characteristics of the primary tumor, with only minor changes in differentiation, expression pattern, and genetic mutation profile.

Cultured primary astrocytes were generated from a slightly injured brain tissue fragment obtained after consent from a patient with cerebral trauma. The grey matter was dissected and dispersed repeatedly after washing in PBS. Primary astrocytes were cultured according to the protocol described by Darling et al [30].

\section{MTT cell growth assay}

To assess the effects of TMZ and/or DMC, $5 \times 10^{3}$ $\mathrm{CD}_{133^{+}} \mathrm{GSCs} /$ well were plated in 96-well plates with six replicate wells at the indicated concentrations of TMZ and/or DMC for 12, 24, 36, 48 and $72 \mathrm{~h}$, respectively. MTT assay was performed to analyze the cell proliferation as described previously [10].

\section{FACS analysis of cell cycle}

$\mathrm{CD}_{133^{+}}$cells that were treated with $\mathrm{TMZ}$ and/or DMC were trypsinized and subsequently fixed with $70 \%$ ice-cold ethanol for $1 \mathrm{~h}$. After PBS washing, the cells were stained in HBSS containing $50 \mu \mathrm{g} / \mathrm{ml}$ Propidium Iodide (PI) (Sigma-Aldrich) and $50 \mu \mathrm{g} / \mathrm{ml}$ of RNase A (Boehringer Mannheim, Indianapolis, IN), for $1 \mathrm{~h}$ at room temperature, and analyzed by FACScan (Becton-Dickinson, San Jose, CA). The cell cycle analysis was performed by ModFit LT software $[11,12]$.

\section{Caspase-3 activity assay}

The Caspase-3 activity was determined using the Caspase-3 activity kit (Beyotime Institute of Biotechnology, Haimen, China). The cells from various samples were homogenized in $100 \mu$ reaction buffer (1\% NP40, 20mMTris-HCl (pH 7.5), 137mM NAD and $10 \%$ glycerol) containing $10 \mu \mathrm{l} 2 \mathrm{mM}$ Ac-DEVD-pNA 
(Caspase- 3 substrate) and incubated at $37^{\circ} \mathrm{C}$ for $2 \mathrm{~h}$. Caspase- 3 activity was measured with an ELISA reader at an absorbance of $405 \mathrm{~nm}$.

\section{TUNEL staining for morphological analysis of apoptosis}

For TUNEL analysis, cells that were treated with DMC and/or ABCG2 shRNA for 24 and 48 h were fixed with $4 \%$ paraformaldehyde for $30 \mathrm{~min}$ before incubating with In Situ Cell Death Detection Kit, Fluorescein (Roche, Palo Alto, CA). Cells were analyzed and enumerated by fluorescence microscopy [13].

\section{ELISA asssays of cell apoptosis}

The Cell Apoptosis ELISA Detection Kit (Roche, Palo Alto, CA) was used to detect Histone-DNA in GSCs after different treatments according to the manufacturer's protocol. Also, the PathScan ${ }^{\circledR}$ Phospho-Stat3 (Tyr705) Sandwich ELISA kit was employed to detect p-STAT3 (Cell Signaling Technology Inc., Danvers, MA) according to the manufacturer's protocol. Briefly, after indicated treatments, cells from different samples were lysed in ice-cold lysis buffer and bound to the immobilized first detection antibody on the ELISA plates. Subsequently, the peroxidase-conjugated secondary antibody was added to each well. After adding the peroxidase substrate, the Histone-DNA and p-STAT3 levels were determined spectrophotmetrically at 405 and $450 \mathrm{~nm}$, respectively.

\section{ROS production detection}

To quantitate ROS in GSCs, cells were loaded with $1 \mu \mathrm{M}$ fluorescent dye dihydrorhodamine for $2 \mathrm{~h}$ before drug treatments. After the drug treatments, GSCs were suspended in PBS on ice, fixed by $70 \%$ Ethanol at $-20^{\circ} \mathrm{C}$ and analyzed by FACS.

\section{In vivo mouse xenograft tumor model}

In vitro cultured $\mathrm{CD} 133^{+} \mathrm{GBM}$ cells $\left(2-5 \times 10^{6}\right.$ in $0.1 \mathrm{ml}$ ) were injected subcutaneously into the flanks of 3-5 week old Balb/c nude mice ( $\mathrm{n}=8 /$ group; 18-22g). After the tumor was established (about $50-75 \mathrm{~mm}^{3}$ ), as determined by caliper measurements, the mice were randomly distributed into the following 4 experimental groups: (a) DMSO control: $100 \mu \mathrm{L}$ DMSO/PBS (1:1) ; (b) TMZ-only: $20 \mathrm{mg} / \mathrm{kg}$ TMZ in $100 \mu \mathrm{L}$ DMSO/PBS; (C) DMC-only: 10 or $30 \mathrm{mg} / \mathrm{kg}$ DMC in $100 \mu \mathrm{L} \mathrm{DMSO} /$ PBS; and (iv) $20 \mathrm{mg} / \mathrm{kg}$ TMZ plus 10 or $30 \mathrm{mg} / \mathrm{kg} \mathrm{DMC}$ in $100 \mu \mathrm{L}$ DMSO/PBS. The treatments were given daily for 24 days. Then, tumor volumes were calculated using the following formula: tumor volume $(\mathrm{TV})=\left(\right.$ length $\times$ width $\left.^{2}\right)$ $\times 0.5$. Based on the absolute tumor volume measurements, we calculated the relative tumor volume (RTV) as RTV = $\mathrm{V}_{\mathrm{t}} / \mathrm{V}_{0}\left(\mathrm{~V}_{0}\right.$, the measured tumor volume at day $0 ; \mathrm{Vt}$, the measured tumor volume at each time point). Further, the relative tumor proliferation rates $(\mathrm{T} / \mathrm{C} \%)$ were calculated using the following formula: $\mathrm{T} / \mathrm{C} \%=\left(\mathrm{T}_{\mathrm{RTV}} / \mathrm{C}_{\mathrm{RTV}}\right) \times 100 \%$, $\left(\mathrm{T}_{\mathrm{RTV}}\right.$ : the treatment group RTV; $\mathrm{C}_{\mathrm{RTV}}$ : model control group RTV). Also, the tumor growth inhibition rates (TGI \%) were calculated using the following formula: Tumor growth inhibition rate $=$ (Average tumor weight of experimental group-Average tumor weight of model group)/Average tumor weight of model group x $100 \%$. All mice procedures were conducted in accordance with the Animal Care guidelines by the First People's Hospital of Kunshan affiliated with Jiangsu University. After 30 days, tumor samples were snap-frozen for further experiments.

\section{Western blot analysis}

For western analysis, GSC cells and tumor samples were solubilized in the protein lysis buffer $(137 \mathrm{mM} \mathrm{NaCl}$, $15 \mathrm{mM}$ EGTA, $0.1 \mathrm{mM}$ sodium orthovanadate, $15 \mathrm{mM}$ $\mathrm{MgCl} 2,0.1 \%$ Triton X-100, 25mM MOPS, $100 \mu \mathrm{M}$ phenylmethylsulfonyl fluoride and $20 \mu \mathrm{M}$ leupeptin, adjusted to $\mathrm{pH}$ 7.2). Then, $30 \mu \mathrm{g}$ protein per sample was subjected to $12 \%$ SDS-PAGE using the Laemmli discontinuous buffer system (Bio-Rad Laboratories, Richmond, CA). The electrophoresed proteins were then transferred to a PVDF membrane and then incubated overnight with anti-human primary antibodies for Cyt c (AC909, Beyotime Biotechnology Inc., Nanjing, China) and ABCG2 (sc-18841, Santa Cruz Biotechnology, Santa Cruz, CA). Then, after 1X TBST washes, the membranes were incubated with the secondary antibodies (A0216, Beyotime Biotechnology Inc., Nanjing, China) for $1 \mathrm{~h}$. The blots were developed with enhanced chemiluminescence (Amersham Life Science, Arlington Heights, IL). For control, the membranes were reprobed with a primary GAPDH antibody (sc-47724, Santa Cruz Biotechnology, Santa Cruz, CA) and further developed.

\section{Statistical analysis}

All statistical tests were performed using SPSS Graduate Pack 11.0 software (SPSS, Chicago, IL). The mean and SE, in addition to one-way ANOVAs were determined to analyze significant differences between groups of data. $\mathrm{P}<0.05$ was considered statistically significant.

\section{ACKNOWLEDGMENTS}

This work was supported by the National Natural Science Foundation of China (CN) (81370062 and 81000963), Jiangsu Province's 333 Talent Program (BRA2011046), Six talents peak project in Jiangsu Province (WSN146), Jiangsu Province's Natural Science Foundation (BK2012670), Medical Research Foundation by Jiangsu Province Health Department (YG201301, 
Z201215 and Z201318), the Clinical Technology Development of Jiangsu University (JLY20120053), and the Brain Clinic and Basic Research Team Program of the First People's Hospital of Kunshan (KYC004). The funding agencies had no role in study design, data collection and analysis, decision to publish, or preparation of the manuscript.

\section{CONFLICTS OF INTEREST}

All authors have declared the sources of research funding for this manuscript and have no financial or other contractual agreements that might cause (or be perceived as causes of) conflicts of interest.

\section{REFERENCES}

1. Stupp R, Tonn JC, Brada M, Pentheroudakis G, ESMO Guidelines Working Group. High-grade malignant glioma: ESMO clinical practice guidelines for diagnosis, treatment and follow-up. Ann Oncol. 2010; 21:v190-3.

2. Athanassiou $\mathrm{H}$, Synodinou $\mathrm{M}$, Maragoudakis E, Paraskevaidis M, Verigos C, Misailidou D, Antonadou D, Saris G, Beroukas K, Karageorgis P. Randomized phase II study of temozolomide and radiotherapy compared with radiotherapy alone in newly diagnosed glioblastoma multiforme. J Clin Oncol. 2005; 23:2372-7.

3. Vescovi AL, Galli R, Reynolds BA. Brain tumour stem cells. Nat Rev Cancer. 2006; 6:425-36.

4. Gong X, Schwartz PH, Linskey ME, Bota DA. Neural stem/ progenitors and glioma stem-like cells have differential sensitivity to chemotherapy. Neurology. 2011; 76:1126-34.

5. Robey RW, Steadman K, Polgar O, Bates SE. ABCG2mediated transport of photosensitizers: potential impact on photodynamic therapy. Cancer Biol Ther. 2005; 4:184-7.

6. Bebes A, Nagy T, Bata-Csörgo Z, Kemény L, Dobozy A, Széll M. Specific inhibition of the ABCG2 transporter could improve the efficacy of photodynamic therapy. J Photochem Photobiol B. 2011; 105:162-6.

7. Jia Q, Zhang X, Deng T, Gao J. Positive correlation of Oct4 and ABCG2 to chemotherapeutic resistance in CD90(+) CD133(+) liver cancer stem cells. Cell Reprogram. 2013; 15:143-50.

8. Phan NL, Trinh NV, Pham PV. Low concentrations of 5-aza-2'-deoxycytidine induce breast cancer stem cell differentiation by triggering tumor suppressor gene expression. Onco Targets Ther. 2015; 9:49-59.

9. Jia Y, Chen J, Zhu H, Jia ZH, Cui MH. Aberrantly elevated redox sensing factor $\mathrm{Nrf} 2$ promotes cancer stem cell survival via enhanced transcriptional regulation of ABCG2 and Bcl-2/Bmi-1 genes. Oncol Rep. 2015; 34:2296-304.

10. Shi L, Wang Z, Sun G, Wan Y, Guo J, Fu X. miR-145 inhibits migration and invasion of glioma stem cells by targeting ABCG2. Neuromolecular Med. 2014; 16:517-28.
11. Xu ZY, Wang K, Li XQ, Chen S, Deng JM, Cheng Y, Wang $\mathrm{ZG}$. The ABCG2 transporter is a key molecular determinant of the efficacy of sonodynamic therapy with Photofrin in glioma stem-like cells. Ultrasonics. 2013; 53:232-8.

12. Jin $Y$, Bin ZQ, Qiang H, Liang C, Hua C, Jun D, Dong WA, Qing L. ABCG2 is related with the grade of glioma and resistance to mitoxantone, a chemotherapeutic drug for glioma. J Cancer Res Clin Oncol. 2009; 135:1369-76.

13. Shi L, Fei X, Wang Z. Demethoxycurcumin was prior to temozolomide on inhibiting proliferation and induced apoptosis of glioblastoma stem cells. Tumour Biol. 2015; 36:7107-19.

14. Shen S, Callaghan D, Juzwik C, Xiong H, Huang $\mathrm{P}$, Zhang W. ABCG2 reduces ROS-mediated toxicity and inflammation: a potential role in Alzheimer's disease. $\mathrm{J}$ Neurochem. 2010; 114:1590-604.

15. Oliva CR, Moellering DR, Gillespie GY, Griguer CE. Acquisition of chemoresistance in gliomas is associated with increased mitochondrial coupling and decreased ROS production. PLoS One. 2011; 6:e24665.

16. Ravindran J, Prasad S, Aggarwal BB. Curcumin and cancer cells: how many ways can curry kill tumor cells selectively? AAPS J. 2009; 11:495-510.

17. Wang W, Zhang L, Wang Z, Yang F, Wang H, Liang T, Wu F, Lan Q, Wang J, Zhao J. A three-gene signature for prognosis in patients with MGMT promoter-methylated glioblastoma. Oncotarget. 2016; 7:69991-9. doi: 10.18632/ oncotarget.11726.

18. Bleau AM, Huse JT, Holland EC. The ABCG2 resistance network of glioblastoma. Cell Cycle. 2009; 8:2936-44.

19. Yodkeeree S, Chaiwangyen W, Garbisa S, Limtrakul P. Curcumin, demethoxycurcumin and bisdemethoxycurcumin differentially inhibit cancer cell invasion through the downregulation of MMPs and uPA. J Nutr Biochem. 2009; 20:87-95.

20. Luthra PM, Kumar R, Prakash A. Demethoxycurcumin induces Bcl-2 mediated G2/M arrest and apoptosis in human glioma U87 cells. Biochem Biophys Res Commun. 2009; 384:420-5.

21. Limtrakul P, Chearwae W, Shukla S, Phisalphong C, Ambudkar SV. Modulation of function of three ABC drug transporters, $\mathrm{P}$-glycoprotein (ABCB1), mitoxantrone resistance protein $(\mathrm{ABCG})$ and multidrug resistance protein 1 (ABCC1) by tetrahydrocurcumin, a major metabolite of curcumin. Mol Cell Biochem. 2007; 296:85-95.

22. Zhang X, Chen Q, Wang Y, Peng W, Cai H. Effects of curcumin on ion channels and transporters. Front Physiol. 2014; 5:94.

23. Shi L, Fei X, Wang Z. Demethoxycurcumin was prior to temozolomide on inhibiting proliferation and induced apoptosis of glioblastoma stem cells. Tumour Biol. 2015; 36:7107-19.

24. Lapidus RG, Carter-Cooper BA, Sadowska M, Choi EY, Wonodi O, Muvarak N, Natarajan K, Pidugu LS, Jaiswal 
A, Toth EA, Rassool FV, Etemadi A, Sausville EA, et al. Hydroxylated dimeric naphthoquinones increase the generation of reactive oxygen species, induce apoptosis of acute myeloid leukemia cells and are not substrates of the multidrug resistance proteins $\mathrm{ABCB} 1$ and $\mathrm{ABCG} 2$. Pharmaceuticals (Basel). 2016; 9:E4. doi: 10.3390/ ph9010004.

25. Buranrat B, Senggunprai L, Prawan A, Kukongviriyapan V. Simvastatin and atorvastatin as inhibitors of proliferation and inducers of apoptosis in human cholangiocarcinoma cells. Life Sci. 2016; 153:41-9.

26. Zhelev Z, Ivanova D, Lazarova D, Aoki I, Bakalova R, Saga T. Docosahexaenoic acid sensitizes leukemia lymphocytes to barasertib and everolimus by ROS-dependent mechanism without affecting the level of ROS and viability of normal lymphocytes. Anticancer Res. 2016; 36:1673-82.
27. Li QC, Liang Y, Tian Y, Hu GR. Arctigenin induces apoptosis in colon cancer cells through ROS/p38MAPK pathway. J BUON. 2016; 21:87-94.

28. Shen S, Callaghan D, Juzwik C, Xiong H, Huang P, Zhang W. ABCG2 reduces ROS-mediated toxicity and inflammation: a potential role in Alzheimer's disease. J Neurochem. 2010; 114:1590-604.

29. Ding Y, Wang H, Niu J, Luo M, Gou Y, Miao L, Zou Z, Cheng Y. Induction of ROS overload by alantolactone prompts oxidative DNA damage and apoptosis in colorectal cancer cells. Int J Mol Sci. 2016; 17:558.

30. Darling JL. The in vitro biology of human brain tumors. In: Thomas DG, editor. Neuro-oncology: primary malignant brain tumors. Baltimore: Johns Hopkins University Press; 1990. p. 1-25. 